\title{
Binding without variables: solving the under-generation problems*
}

\author{
Yimei Xiang \\ Rutgers University
}

\begin{abstract}
The variable-free semantics of Jacobson (1999, 2000, 2014) derives binding relations by a locally applied type-shifting rule called ' $z$ '. This rule, however, under-generates binding. To solve this problem, I replace the z-rule with the dependency-generating rule ' $i$ ', which applies to predicates that contain a bindable pronoun. Furthermore, to account for the interactions between scoping and binding, I enrich the variable-free system by combining it with a two-dimensional analysis of quantifiers. Topics covered in this paper include: binding into adjuncts, possessor binding, scope ambiguity, inverse linking, weak crossover, and 'paycheck pronouns'.
\end{abstract}

Keywords: pronouns, binding, variable-free, direct compositionality, non-c-commanding binding, scoping, inverse linking, weak crossover, paycheck pronouns, categorial grammar

\section{Introduction}

\subsection{Jacobson's variable-free approach}

Jacobson's (1999; 2000; 2014) variable-free semantics assumes no index/variable in the grammar. ${ }^{1}$ In contrast to 'variable-full' approaches, which translate pronouns into variables and interpret these variables via assignment functions (as in Kratzer \& Heim 1998), 'variable-free' approaches analyze pronouns as instances of the category $\mathrm{NP}^{\mathrm{NP}}$ that denote identity functions over entities, as exemplified in (1).

$$
\mathrm{CAT}(i t)=\mathrm{NP}^{\mathrm{NP}}, \text { and } \llbracket i t \rrbracket=\lambda x_{e} \cdot x
$$

More generally, as exemplified in (2-ii), pronouns and expressions that contain an unbound pronoun are referred to as ' $\mathrm{X}^{\mathrm{NP}}$-categories', represented with a 'sup(erscript)-NP abstraction'. $\mathrm{X}^{\mathrm{NP}}$ has the same semantics as the functional counterpart $\mathrm{X} \mid \mathrm{NP}$, which has a 'slash-NP abstraction' ; 2 however, in contrast to $\mathrm{X} \mid \mathrm{NP}, \mathrm{X}^{\mathrm{NP}}$

* For helpful discussions, I thank Simon Charlow, Michael Glanzberg, Pauline Jacobson, Haoze Li, Ken Safir, and the abstract reviewers and the audience of SALT 31. All errors are mine.

1 The variable-free hypothesis is widely assumed in works on Categorial Grammar. The system presented in this paper is developed from a series work by Jacobson beginning in the late 1980s.

2 I use ' $A \mid B$ ' as the joint representation for $A / B$ and $A \backslash B$, which select a $B$ argument on the right and on 
doesn't actively merge with an NP in syntax. For example, their mom has the same semantics as _'s mom, but it doesn't take an NP argument.

\begin{tabular}{rr|lr} 
& & Category & Meaning \\
\hline (i) & their mom & $\mathrm{NP} N \mathrm{NP}$ & $\lambda x_{e} \cdot \operatorname{mom}(x)$ \\
(ii) & 's mom & $\mathrm{NP} \backslash \mathrm{NP}$ & $\lambda x_{e} \cdot \operatorname{mom}(x)$ \\
\hline (i) & invited him & $(\mathrm{S} \backslash \mathrm{NP})^{\mathrm{NP}}$ & $\lambda y_{e} \lambda x_{e} . i v t(x, y)$ \\
(ii) & invited & $(\mathrm{S} \backslash \mathrm{NP}) / \mathrm{NP}$ & $\lambda y_{e} \lambda x_{e} . i v t(x, y)$
\end{tabular}

To compose expressions with pronouns, Jacobson assumes a type-shifting rule g-sup(script), defined as in (3). Applying g-sup to a functional expression $\alpha$ that selects an $\mathrm{X}$ argument allows this $\alpha$ to merge with an $\mathrm{X}^{\mathrm{NP}}$ argument, and further, it passes up the information about the unbound pronoun contained in this $\mathrm{X}^{\mathrm{NP}}$.

(3) The g-sup-rule (for passing up info about unbound pronouns)

For any expression $\alpha$ s.t. $\operatorname{CAT}(\alpha)=A \mid B$, we have:

$$
\operatorname{CAT}(\mathrm{g}-\sup (\alpha))=A^{C} \mid B^{C} \text {, and } \llbracket \mathrm{g}-\sup (\alpha) \rrbracket=\lambda V_{c b} \lambda C_{c} \cdot \llbracket \alpha \rrbracket(V(C))
$$

Consider (4) for example. The verb invited, which selects an NP object, cannot directly apply to the pronominal object his mom, which is an NPNP; however, the type-shifted verb g-sup (invited) can apply to an NPNP. The resulting VP inherits the sup-NP abstraction, indicating that the pronoun remains unbound in this VP.

Composition of invited his mom (without binding) ${ }^{3}$

$$
\begin{aligned}
& \text { invited } \\
& (\overline{\mathrm{S}} \backslash \mathrm{NP}) / \mathrm{NP} \quad \lambda y_{e} \lambda x_{e} \cdot \overline{i v t}(\bar{x}, y)
\end{aligned}
$$

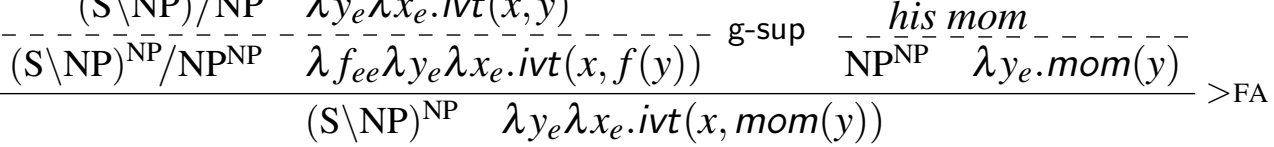

The g-sup rule is analogous to the so-called 'Geach rule' (Geach 1970), referred to as ' $g$-sl(ash)' in Jacobson's system. These two g-rules have the same effects in semantics, but g-sl is used to pass up information about an unsaturated syntactic argument. For example, in didn't come, applying g-sl to the negative auxiliary didn't shifts it from an $\mathrm{S} / \mathrm{S}$ to an $(\mathrm{S} \backslash \mathrm{NP}) /(\mathrm{S} \backslash \mathrm{NP})$, which can merge with the intransitive verb come. The result of composition is an $S \backslash N P$, where the backslash-NP abstraction indicates that the subject argument remains unsaturated.

(5) The g-sl-rule (for passing up info about unsaturated syntactic arguments)

For any expression $\alpha$ s.t. $\operatorname{CAT}(\alpha)=A \mid B$, we have:

$$
\mathrm{CAT}(\mathrm{g}-\mathrm{sl}(\alpha))=(A / C) \mid(B / C) \text {, and } \llbracket \mathrm{g}-\mathrm{sl}(\alpha) \rrbracket=\lambda V_{c b} \lambda C_{c} \cdot \llbracket \alpha \rrbracket(V(C))
$$

the left, respectively, and return an $A$. (Jacobson writes them as ' $A /{ }_{\mathrm{R}} B$ ' and ' $A /{ }_{\mathrm{L}} B$ '.) I also use ' $A \ddagger B$ ' as the joint representation for categories corresponding to the type $\langle\operatorname{TYPE}(B), \operatorname{TY} \operatorname{PE}(A)\rangle$, including $A / B, A \backslash B, A^{B}$, and $A[B$.

3 In the derivation graphs, dotted lines are for unary rules, such as $g$ and $z$; full lines are for binary rules, such as forward Functional Application $(>\mathrm{FA})$ and backward Functional Application $(<\mathrm{FA})$. 
Binding without variables: solving the under-generation problems

Further, to tackle sentences with multiple unbound pronouns, Jacobson (1999) proposes to define g-sup recursively. In consequence, applying g-sup $n$ to a functional expression $\alpha$ has the effect of applying g-sup to $\alpha$ while skipping $n$-many abstractions in $\alpha$. The following definition also extends this idea to g-sl:

The recursive g-rules (after Jacobson 1999)

a. For any meaning $F$ of type $\langle a, b\rangle, \mathrm{g}_{0}(F)=\lambda V_{c b} \lambda C_{c} . F(V(C))$.

b. For any expression $\alpha$ s.t. $\operatorname{CAT}(\alpha)=(A \mid B) \ddagger \vec{X}^{n}$, we have:

i. $\operatorname{CAT}\left(g-\sup _{n}(\alpha)\right)=\left(A^{C} \mid B^{C}\right) \ddagger \vec{X}^{n}$

ii. $\operatorname{CAT}\left(\mathrm{g}-\mathrm{Sl}_{n}(\alpha)\right)=((A / C) \mid(B / C)) \ddagger \vec{X}^{n}$

iii. $\llbracket g-\sup _{n}(\alpha) \rrbracket=\llbracket g-\mathrm{s}_{n}(\alpha) \rrbracket=\lambda D \cdot \mathrm{g}_{n-1}(\llbracket \alpha \rrbracket(D))$

Derivation (7) exemplifies recursive g-sup. As noted, a VP (of category $S \backslash N P$ ) needs to be type-shifted by g-sup when it merges with a pronominal subject. The basic g-sup rule defined in (3) doesn't apply to the VP in (7), because this VP has an extra sup-NP abstraction stemming from the possessive pronoun his. Defining g-sup recursively overcomes this problem: applying g-sup 1 means saturating the extra NP abstraction temporarily, applying g-sup as normal, and then abstracting the NP back.

$$
\begin{aligned}
& \text { She } e_{i} \text { invited his j mom. (Unbound interpretation) }
\end{aligned}
$$

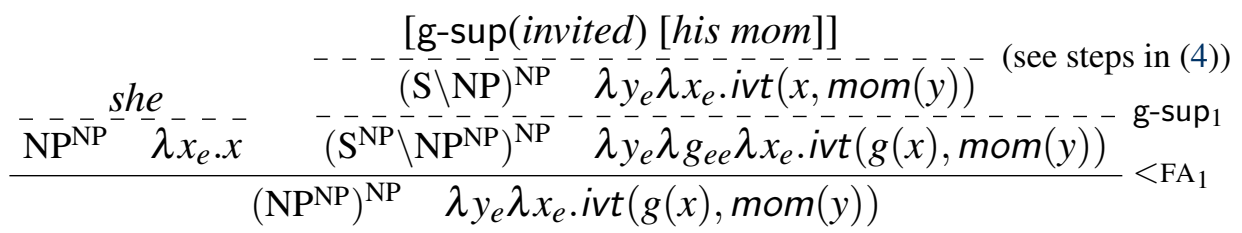

Analogously, following a suggestion from Pauline Jacobson (p.c.), I assume that the g-sup ${ }_{1}$-shifted VP composes with the subject argument via 'generalized' backward FA $\left(<\mathrm{FA}_{1}\right)$, which has the effect of applying g-sup ${ }_{1}(\mathrm{VP})$ to the subject while skipping the sup-NP abstraction in the VP stemming from the pronoun it contains. ${ }^{4}$

4 Instead of generalizing binary composition rules or defining them recursively, Jacobson 1999 assumes that the composition between an NP and a pronoun-containing VP involves generalized Montague lift. For (7), type-lifting the subject she allows it to take the VP as an argument.

As Pauline Jacobson (p.c.) points out, however, such composition can be easier if composition rules are recursive or generalized, just like g. I assume a generalized definition of backward FA as in (i). Here $\lambda \vec{x}^{n}$ denotes a sequence of abstractions stemming from the $n$-many unbound pronouns in $\alpha$, and $\lambda \vec{y}^{m}$ denotes a sequence of abstractions corresponding to the $m$-many unsaturated syntactic arguments of $\alpha$. The application of $<\mathrm{FA}_{n+m}$ to $[\beta-\alpha]$ says: apply $\alpha$ to $\beta$ while skipping the $n$-many pronominal abstractions in $\alpha$ as well as the $m$-many abstractions of $\alpha$ that should be filled by strings appearing on the right of $\alpha$. Other binary composition rules can be generalized analogously.

(i) $\quad$ For any expressions $\alpha$ and $\beta$ s.t. $\operatorname{CAT}(\alpha)=\left((A \backslash B) / \vec{Y}^{m}\right)^{\vec{X}^{n}}$ and $\operatorname{CAT}(\beta)=B$, we have:

$$
\operatorname{CAT}([\beta-\alpha])=\left(A / \vec{Y}^{m}\right)^{\vec{X}^{n}} \text {, and } \llbracket \beta-\alpha \rrbracket \underset{<\mathrm{FA}_{n+m}}{\longrightarrow} \lambda \vec{x}^{n} \lambda \vec{y}^{m} \cdot \llbracket \alpha \rrbracket\left(\vec{x}^{n}\right)\left(\vec{y}^{m}\right)(\llbracket \beta \rrbracket) \text {. }
$$


For binding, Jacobson assumes that pronoun binding is achieved by the locally applied rule ' $z$ ', defined as in (8). Applying the z-rule to a transitive verb closes off the dependency between the two arguments of this verb. Then binding arises when this z-shifted verb merges with a string that contains the bindee and the binder.

The z-rule (to create binding relations)

For any expression $\alpha$ s.t. CAT $(\alpha)=(A \backslash \mathrm{NP}) / B$, we have:

$\operatorname{CAT}(\mathrm{z}(\alpha))=(A \backslash \mathrm{NP}) / B^{\mathrm{NP}}$, and $\llbracket \mathrm{z}(\alpha) \rrbracket=\lambda f_{e b} \lambda x_{e} \cdot \llbracket \alpha \rrbracket(f(x))(x)$.

As seen in (9), the type-shifted verb z(invited), interpreted as $\lambda f_{e e} \lambda x_{e}$.ivt $(x, f(x))$, is already encoded with a co-argument dependency. Composing this verb with its two arguments by FA yields an S read as 'John invited John's mom'.

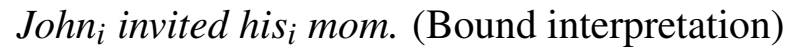

$$
\begin{aligned}
& \text { _. }
\end{aligned}
$$

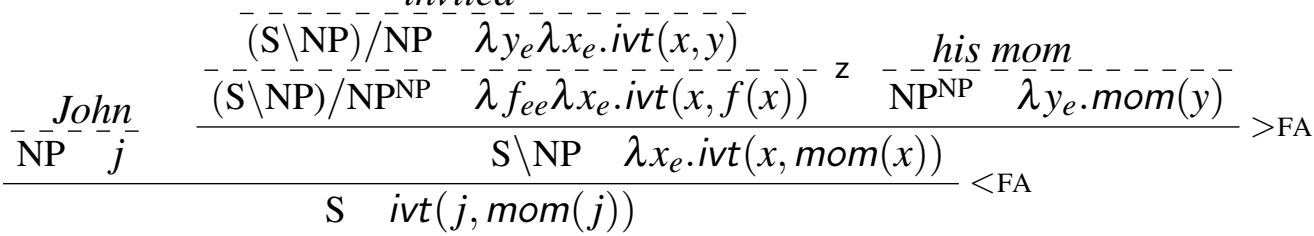

\subsection{Problems of under-generation}

Example (9) is an instance of co-argument binding. In co-argument binding, the bindee appears within a lower argument of the z-shifted functional expression, and the binder serves as a higher argument of this expression by itself.

$$
\text { Schema of co-argument binding: } \quad \text { [[BINDER] z(verb) [... BINDEE ...]] }
$$

However, there are many cases where the bindee and the binder do not stand in a configuration like (10). First, as seen in (11), the bindee may occur in an adjunct, not an argument. (For Jacobson's solution, see fn. 7; for more data, see Barker 2012.)

(11) a. Mary $i$ went home [with her ${ }_{i}$ mom].

b. We will sell no wine [before $_{i}$ its $_{i}$ time].

Second, the binder itself may not be an argument of a functional expression. For example in (12a), the binder is the possessor John, not the entire subject John's mother. In the Government-and-Binding (GB) theory, such binding cases are called 'non-c-commanding binding', which include binding out of DPs, as in (12a,b), and binding out of adjuncts, as in (12c). ${ }^{5}$ (For more data, see Barker 2012.)

a. $\left[\mathrm{John}_{i}\right.$ 's/ His ${ }_{i} /$ Every boy ${ }_{i}$ 's mother] loves him ${ }_{i}$.

5 I use '(non-)c-commanding' only for description. To be exact, Jacobson's system doesn't have the concept of c-commanding, because this system doesn't model syntax in terms of tree structures. 
Binding without variables: solving the under-generation problems

b. [Someone from every city ${ }_{i}$ ] despises $i_{i}$.

c. [In everyone ${ }_{i}$ 's own mind], they ${ }_{i}$ are the most important person.

The primary goal of this paper is to solve the binding under-generation problems while keeping the basic apparatus of Jacobson's variable-free system. Moreover, I will enrich this system to account for the interactions between scoping and binding.

\section{Proposal: binding by the i-rule}

For binding in general, I propose to replace Jacobson's z-rule with the i-rule defined in (13) (a la the duplicator W in Szabolcsi 1992). ${ }^{6}$ The i-rule has an 'identity' effect: for any multi-ary predicate $\alpha$ that contains a bindable pronoun, applying i to $\alpha$ has the consequence that whatever saturates the sup-NP abstraction stemming from the bindable pronoun contained in $\alpha$ also saturates the next NP abstraction of $\alpha$.

(13) The i-rule (for generating dependencies)

For any expression $\alpha$ s.t. $\operatorname{CAT}(\alpha)=(A \ddagger \mathrm{NP})^{\mathrm{NP}}$, we have:

$\mathrm{CAT}(\mathrm{i}(\alpha))=A \ddagger \mathrm{NP}$, and $\llbracket \mathrm{i}(\alpha) \rrbracket=\lambda x_{e} \cdot \llbracket \alpha \rrbracket(x)(x)$.

The proposed i-rule and Jacobson's z-rule stand in the following relation:

(14) For any transitive verb $F$ and $\mathrm{NP}^{\mathrm{NP}}$-category $f$, i $([\mathrm{g}$-sup $(F)](f))$ is identical to $[z(F)](f)$ in both syntax and semantics.

Consider (15b) for illustration. First, composing g-sup(invited) with his mom returns a VP with two NP abstractions, one sup-NP $(\lambda y)$ from the pronoun his, and one slash-NP $(\lambda x)$ from the unsaturated subject. Second, applying i to the resulting VP makes the two NP abstractions be bound by the same abstractor, reducing it into a one-place predicate with a dependency on the subject: ' $\lambda x_{e}: x$ invited $x$ 's mom.'

(15) Ways to derive the bound interpretation of invited his mom:

a. By the z-rule (as in (9)):

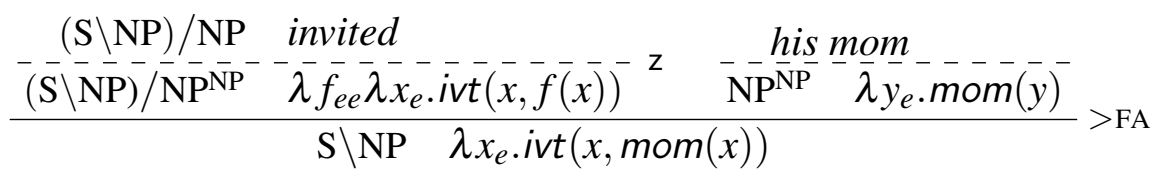

b. By the i-rule:

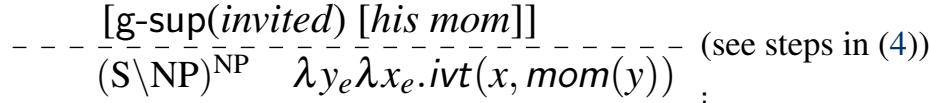

$$
\begin{aligned}
& \mathrm{S} \backslash \mathrm{NP} \quad \lambda x_{e} \cdot \operatorname{ivt}(x, \operatorname{mom}(x))
\end{aligned}
$$

6 The i-rule is assumed in light of the duplicator $\mathrm{W}(:=\lambda F \lambda x . F(x)(x))$ in Combinatory Categorial Grammar. Szabolcsi (1992) takes this duplicator to be the lexicon of a reflexive pronoun like herself; Shan \& Barker (2006) and Charlow (To appear) build the duplicator into a binding rule that applies to quantificational binders. 
As seen above, the z-rule is locally applied to the transitive verb invited; in contrast, the i-rule is applied after the verb has merged with a bindee-containing expression.

Example (15) has shown how the i-rule accounts for co-argument binding due to the equivalence relation in (14), the proposed i-rule, together with the existing rule g-sup, can account for any binding relations that can be derived by the z-rule. Moreover, as I will argue below, the i-rule captures several other binding relations.

The i-rule allows for binding into adjuncts. It is applied after the verb has composed with the bindee-containing expression, no matter whether this expression is an argument of the verb or an adjunct of a VP. In (16), option (16a) is deviant: the type-shifted verb $\mathrm{z}$ (invited) selects an $\mathrm{NP}^{\mathrm{NP}}$-argument, and thus it cannot merge

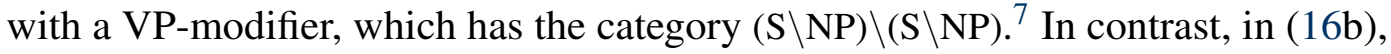
applying the i-rule to the PP-modified VP left with his mom derives a dependency between the subject argument and the pronoun contained within the PP-adjunct.

$\mathrm{John}_{i}$ left [with his $i$ mom].
a. * John z(left) [with his mom]
b. John i [left [with his mom]]
c. i [LIFT(John) left [with his mom]]

Option (16c) will be discussed in section 3.3: by type-lifting the binder John into a quantifier, which will be analyzed as a two-dimensional expression with a 'trace'-like component, we can derive the dependency by applying i to the entire sentence.

The i-rule also sufficiently accounts for pronominal binding, regardless of 'ccommanding' - all we need is to apply the i-rule to an expression that contains both the bindee and the pronominal binder. (17) is a case of 'non-c-commanding' pronominal binding, where the pronominal possessor within the subject binds the object pronoun. The z-rule cannot capture this binding relation: in (17a), z-shifting the verb loves would make the object be bound by the entire subject.

7 Jacobson derives binding into adjuncts by applying generalized Montague lift (et-LIFT) to the VP, which allows this VP to take a VP-adjunct as an argument. The analysis precedes as follows:

(i) $\quad[$ John z(et-LIFT(left)) [with his mom $]]$

First, applying et-LIFT to the VP left shifts it into an (S $\backslash N P) /((S \backslash N P) \backslash(S \backslash N P))$, which can take the PP-adjunct (of category (S $\backslash \mathrm{NP}) \backslash(\mathrm{S} \backslash \mathrm{NP})$ ) as an argument. Second, applying $\mathrm{z}$ to the type-lifted VP closes off the dependency between the two argument slots that will be saturated by the subject and the adjunct. Last, the resulting VP merges with the bindee-containing adjunct and the subject binder. (See Jacobson 1999 for a related discussion tackling unbound readings.) Despite the distinct analytical moves, the analyses in (16b) and in (i) converge on the idea that binding is independent of grammatical distinctions between arguments and adjuncts.

However, as pointed out by Simon Charlow (p.c.), the generalized-lifting analysis in (i) has limitations; it doesn't extend to cases where the binder is inside the VP, such as in We will sell no wine $_{i}$ before its $_{i}$ time. For my own explanation, see section 3.3. 
Binding without variables: solving the under-generation problems

(17) $\left[\mathrm{His}_{i}\right.$ mom] loves $\operatorname{him}_{i}$.
a. \# [[His mom] z(loves) him]
(Meaning: 'His mom loves his mom.' $\times$ )
b. [i (His mom loves him) ]
(Meaning: 'His mom loves him.' $\sqrt{ }$ )

More details of (17b) are given below. Due to the two pronominal expressions it contains, the sentence is first analyzed as an $\left(\mathrm{S}^{\mathrm{NP}}\right)^{\mathrm{NP}}$. The composition involves gsup $_{1}$ and $F_{1}$, just as in (7). Next, applying i to the full sentence yields a dependency between his and him, reducing the sentence into an $\mathrm{S}^{\mathrm{NP}}$.

$$
\begin{aligned}
& \text { loves him }
\end{aligned}
$$

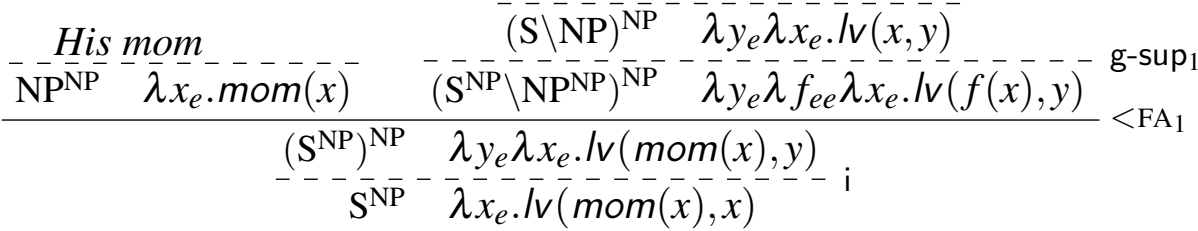

\section{Extension: quantificational binding}

In many cases, scoping feeds 'non-c-commanding' binding (Safir 2004; Barker 2005, 2012; a.o.). These cases argue that quantificational binding is available only if the quantificational binder scopes above the bindee. Since Jacobson's system doesn't deal with scoping, the relation between scoping and binding remains a mystery.
a. [Every boy ${ }_{i}$ 's mother] loves $\operatorname{him}_{i}$.
(Possessor binding)
b. [Someone from every city $_{i}$ ] despises it $_{i}$.
(Inverse linking)

In GB-style compositional semantics, semantic binding is achieved by making the pronoun co-indexed with the trace of the quantifier at LF (Kratzer \& Heim 1998). As in (20), the trace of the quantifier and the bound pronoun both carry the index $i$ :

$$
\text { [ every-boy } \lambda i\left[t_{i} \text { loves his }_{i}\right. \text { mother ]] }
$$

In this section, I will propose a variable-free approach to quantificational binding. My approach takes insight from (20) but does not make use of quantifier raising or indexation. ${ }^{8}$ Briefly, by applying the i-rule, my approach has the effect of generating a dependency between a 'trace'-like component of the quantifier and the pronoun.

8 In GB-style compositional semantics, semantic composition takes place at LF, an abstract level of representations in which the linear order of strings can be different from that in the surface structure. In contrast, Jacobson assumes 'Direct Compositionality' (Barker \& Jacobson 2007), a hypothesis independent of the variable-free hypothesis. She argues that syntax and semantics work in tandem: "Each linguistic expression that is proven well-formed in the syntax is assigned a meaning by the semantics, and the syntactic rules or principles which prove an expression as well-formed are paired with the semantics which assign the expression a meaning." (Jacobson 2014: p. 9). As a result, this system has no intermediate level like LF and no transformation (e.g., LF movement) for mapping surface representations into LF representations. For this consideration, my analysis of scoping doesn't resort to quantifier raising. 


\subsection{Quantificational DPs as two-dimensional expressions}

I assume that quantificational DPs are two-dimensional expressions (cf. 'Quantifier Storage' in Cooper 1983), represented in the form of $\left[\frac{\text { тор }}{\text { вотто }}\right]$. For example, the category and meaning of everyone are as follows (eo abbreviates $\lambda P_{e t}$.eo $\left(\lambda x_{e} . P(x)\right)$ ):

$$
\text { a. } \mathrm{CAT}(\text { everyone })=\left[\frac{\mathrm{S}[(\mathrm{S} / \mathrm{NP})}{\mathrm{NP} / \mathrm{NP}}\right]\left(\text { abbr.: }\left[\frac{\Pi}{T}\right]\right) \quad \text { b. } \llbracket \text { everyone } \rrbracket=\left[\frac{e o}{\lambda x_{e} \cdot x}\right]
$$

The bottom component is 'trace'-like. ${ }^{9}$ It is interpreted as an identity function over entities, just like pronouns; however, in syntax, it has a distinct category NP/NP (abbreviated as $T$ ), which applies to an NP argument on the right and returns an NP. The top component has the category $\mathrm{S}[(\mathrm{S} / \mathrm{NP})$ (abbreviated as $\Pi$ ) and is interpreted as a generalized quantifier. I assume that the two components have distinct roles in composition. The bottom component participates in composition and binding, similar to the assumption in the GB-theory that it is the trace of the quantificational binder that composes with the surrounding expressions and is co-indexed with the bindable pronouns. The top component deals with quantification and scoping; it remains unchanged till it can combine with the bottom component via 'inward FA'.

To tackle composition involving two-dimensional expressions, I define binary FA rules as in (22). (22a) says: when a two-dimensional expression $\alpha$ composes with a one-dimensional expression by FA, it is the bottom component of $\alpha$ that participates in the reduction by FA. (22b) tackle cases with multiple quantifiers: if both of the expressions that participate in FA are two-dimensional, the one that serves as the function takes narrow scope. Other binary rules are defined analogously.

Forward FA (backward FA is analogous)

$$
\begin{aligned}
& \text { a. }\left(\mathrm{A} / \mathrm{B}\left[\frac{\mathrm{X}}{\mathrm{B}}\right]\right) \text { or }\left(\left[\frac{\mathrm{X}}{\mathrm{A} / \mathrm{B}}\right] \mathrm{B}\right) \underset{>\mathrm{FA}}{\longrightarrow}\left[\frac{\mathrm{X}}{\mathrm{A}}\right] \\
& \text { b. }\left(\left[\frac{\mathrm{X} 1}{\mathrm{~A} / \mathrm{B}}\right]\left[\frac{\mathrm{X} 2}{\mathrm{~B}}\right]\right) \underset{>\mathrm{FA}}{\longrightarrow}\left[\frac{\mathrm{X} 2}{\left(\left[\frac{\mathrm{X} 1}{\mathrm{~A} / \mathrm{B}}\right] \mathrm{B}\right)>\mathrm{FA}}\right]=\left[\frac{\mathrm{X} 2}{\left[\frac{\mathrm{X} 1}{\mathrm{~A}}\right]}\right]
\end{aligned}
$$

Inward FA applies to a single two-dimensional expression. As schematized in (23a), when the bottom component of a two-dimensional expression can serve as the argument of the top, apply the top to the bottom and reduce this two-dimensional

9 To be exact, Jacobson's framework doesn't assume empty categories like traces in grammar. However, assuming traces doesn't affect composition; for example, assume that a trace $t$ is an NP/NP-category denoting an identity function, then the VP [g-sl(invite) $t$ ], which contains an object trace, is equivalent to the transitive verb invite, in both syntax and semantics. In this paper, saying that an expression has a 'trace'-like component means that this expression contains an unfilled non-subject argument, a two-dimensional quantifier, or some other $T$-category. 
Binding without variables: solving the under-generation problems

expression into a one-dimensional expression. Further, (23b) says: for a recursion of top-bottom pairs, the effect of inward FA applies to the innermost top-bottom pair.

(23) Inward FA (a la lower in Shan \& Barker 2006 and Barker \& Shan 2014)

a. $\left[\frac{\mathrm{A}[\mathrm{B}}{\mathrm{B}}\right] \underset{\mathrm{VFA}}{\longrightarrow} \mathrm{A} \quad$ b. $\left[\frac{\mathrm{C}[\mathrm{A}}{\left[\frac{\mathrm{A}[\mathrm{B}]}{\mathrm{B}}\right]}\right] \underset{\mathrm{VFA}}{\longrightarrow}\left[\frac{\mathrm{C}[\mathrm{A}}{\left(\left[\frac{\mathrm{A}[\mathrm{B}] V_{\mathrm{FA}}}{\mathrm{B}}\right]\right.}\right]=\left[\frac{\mathrm{C}[\mathrm{A}}{\mathrm{A}}\right] \underset{\mathrm{VFA}}{\longrightarrow} \mathrm{C}$

When a unary rule that is defined for one-dimensional expressions (e.g., g, i, and LIFT) is applied to a two-dimensional expression, effects of this rule apply to the bottom component of this expression, as schematized in (24).

If $\mathbb{R}$ is a unary rule defined for one-dimensional expressions, we have:

$$
\left[\frac{\mathrm{A}[\mathrm{B}}{\mathrm{C}}\right] \underset{\mathbb{R}}{\rightarrow}\left[\frac{\mathrm{A}[\mathrm{B}}{\mathbb{R}(\mathrm{C})}\right]
$$

In a stack of top-bottom pairs, (24) applies recursively; as a result, effects of a unary rule apply to the bottom component of the innermost top-bottom pair in the stack.

\section{2 (Non-)quantificational possessor binding}

With the assumptions made for quantificational DPs, the analysis of pronominal possessor binding easily extends to quantificational possessor binding. In (25b), the binder role of the pronominal possessor in (25a) (of category $\mathrm{NP}^{\mathrm{NP}}$ ) is now taken by the 'trace'-like component of the quantificational possessor (of category NP/NP).
a. His ${ }_{i}$ mom loves $\operatorname{him}_{i}$.
(Pronominal possessor binding)
b. Every boy's mom loves $\operatorname{him}_{i}$.
(Quantificational possessor binding)
c. John ${ }_{i}$ 's mother loves $\operatorname{him}_{i}$.
(Non-quantificational possessor binding)

The composition of (25b) precedes as in (26). First, applying g-sl and g-sl ${ }_{1}$ to _'s mom and loves him respectively allows them to select a $T$. Second, the 'trace'-part of every boy composes with the remnant DP _'s mom and then with the VP loves him via backward FA, returning an $(\mathrm{S} / \mathrm{NP})^{\mathrm{NP}}$-category denoting a two-place predicate. In this output, the sup-NP comes from the bindee him, and the right-slash NP comes from the 'trace'-part of the quantificational binder every boy. Third, applying i to the resulting two-dimensional sentence yields a dependency between these two NPs and shifts the bottom component of the sentence into an S/NP denoting a one-place predicate. This S/NP may serve as an argument of the quantificational part at the top. (Recall that $\Pi$ abbreviates $S[(\mathrm{~S} / \mathrm{NP})$.) Finally, applying inward FA reduces the two-dimensional sentence into a simple $\mathrm{S}$ expressing quantificational binding: 'For every boy $x, x$ 's mom loves $x$.'

(26) Every boy ${ }_{i}$ 's mom loves $\operatorname{him}_{i}$. 


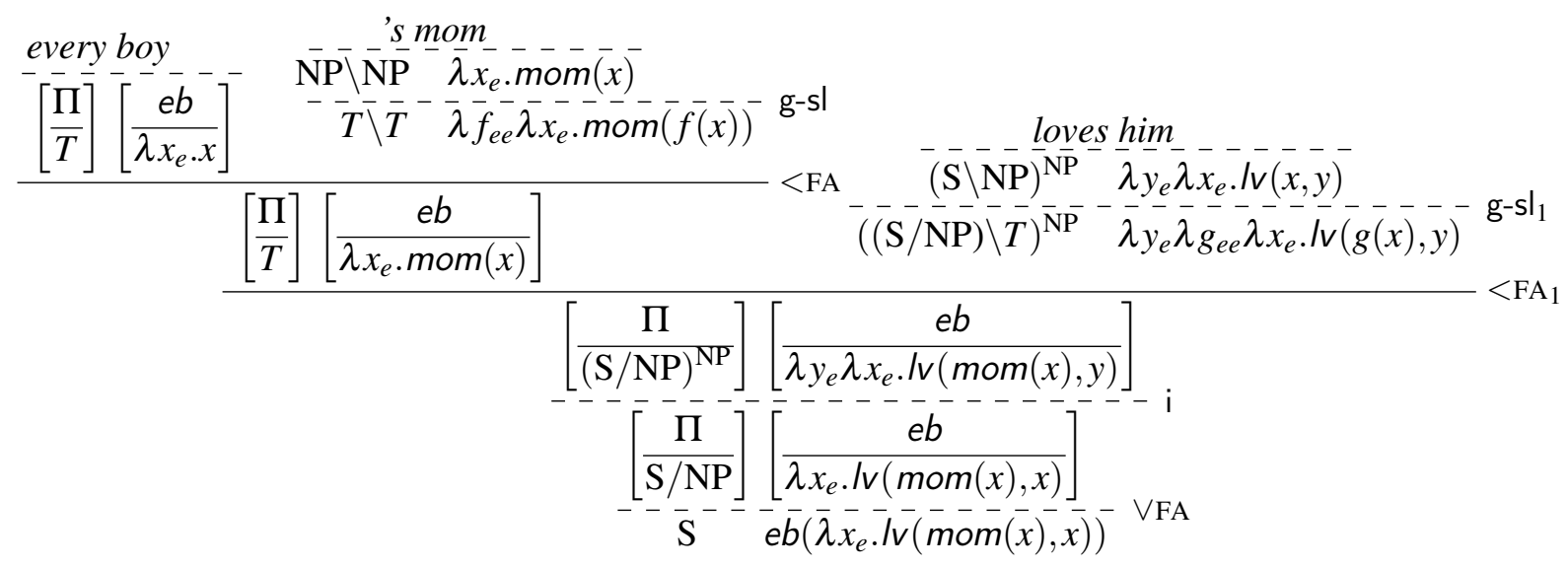

This analysis also extends to the non-quantificational possessor binding case (25c). All we need is to lift the binder John into a quantifier by the rule in (27).

(27) Lift (a la Montague lift)

For any expression $\alpha$ s.t. $\operatorname{CAT}(\alpha)=\mathrm{NP}$, we have:

$$
\operatorname{CAT}(\operatorname{LIFT}(\alpha))=\left[\frac{\mathrm{S}[(\mathrm{S} / \mathrm{NP})}{\mathrm{NP} / \mathrm{NP}}\right]\left(\text { abbr.: }\left[\frac{\Pi}{T}\right]\right) \text {, and } \llbracket \operatorname{LIFT}(\alpha) \rrbracket=\left[\frac{\lambda P_{e t} \cdot P(\llbracket \alpha \rrbracket)}{\lambda x_{e} \cdot x}\right] \text {. }
$$

\subsection{More cases of binding into adjuncts}

The analysis of binding into adjuncts given in (16b) works only if the bindee depends on the subject and is contained within the (modified) VP. It doesn't apply to the cases below: in (28a), the binder no wine is inside the VP; in (28b), the if-clause modifies a sentence, not a VP. These cases are also difficult to Jacobson's solution (see fn. 7).

a. We will sell no wine [before its $_{i}$ time].

b. [ $\mathrm{John}_{i}$ will be disappointed] [if his ${ }_{i}$ mom is late].

The two-dimensional account now allows us to derive dependencies in the above cases by the i-rule. Basically, the analysis in (16b) allows the i-rule to be applied to a string that contains the bindee but not the binder; here, by analyzing the binder as a two-dimensional expression, we can apply the i-rule to a string that contains also the binder, no matter whether this string is a VP or a sentence.

For example, in (28a), applying g-sup to each of the strings that 'c-command' its passes up the information about this bindable pronoun; likewise, applying gsl to the strings that 'c-command' the object no wine passes up the information about the 'trace'-part of this quantificational binder. Composition yields a twodimensional sentence with an $(\mathrm{S} / \mathrm{NP})^{\mathrm{NP}}$-category at the bottom. Further, applying $i$ to this sentence closes off the dependency between its and the 'trace'-part of no wine, yielding a binding-into-adjunct effect. 
Binding without variables: solving the under-generation problems

\subsection{Scope ambiguity}

In GB-style compositional semantics, scope ambiguity of quantifiers is derived by quantifier raising. In the enriched variable-free system proposed in this paper, as seen in the definition of FA in (22b), the scopal relation between two quantifiers is determined by how they participate in FA:

(29) For a sentence S of the form "Q1 V Q2", where Q1 and Q2 are quantificational DPs, we have: (i) $S$ has a surface scope reading if the bottom component of Q1 serves as an argument of [V Q2]; (ii) $S$ has an inverse scope reading if $\mathrm{Q} 1$ as a whole serves as an argument of [V Q2].

\subsubsection{Surface scoping}

As illustrated in Figure 1, composing the subject everyone with the VP loves someone by backward FA derives the surface scope reading, where eo scopes above so in (a). The resulting stack is reduced into a simple $\mathrm{S}$ by applying inward FA twice.

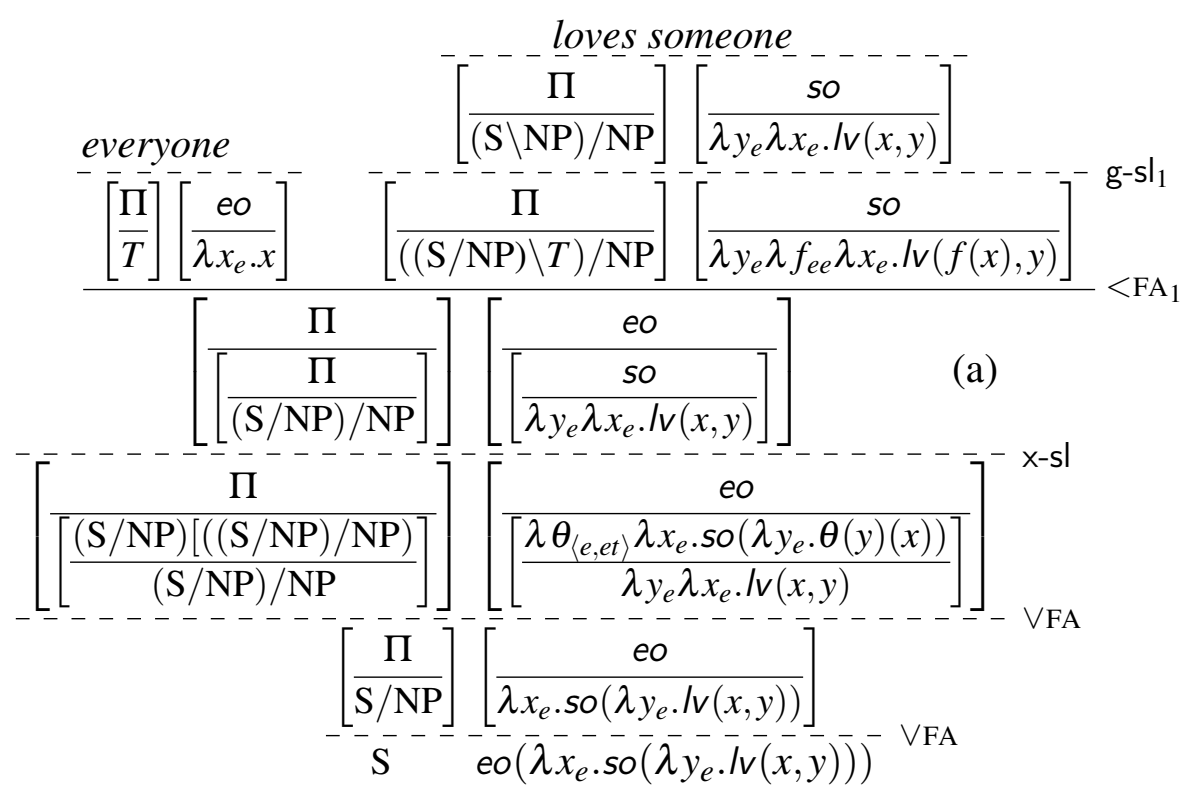

Figure 1 Surface scope reading of Everyone loves someone

One caveat here is that inward FA doesn't directly apply to (a) in Figure 1: the quantificational part of someone should combine with an S/NP, but here the bottom component has the category (S/NP)/NP, which contains an extra slashNP abstraction (corresponding to $\lambda x$ ) stemming from the 'trace'-part of everyone. 
I resolve this type-mismatch by the following x-rules, which apply to particular two-dimensional expressions (here the subscript $c$ in $\mathbf{x}_{c}$ refers to the category $C$ ):

$$
\begin{aligned}
& \text { The x-rules } \\
& \text { For any two-dimensional expression }\left[\frac{\alpha}{\beta}\right] \text { s.t. } \\
& \operatorname{CAT}(\alpha)=A[(B \ddagger X) \text { and } \operatorname{CAT}(\beta)=(B / C) \ddagger X \text {, we have: } \\
& \text { x-sl }\left(\left[\frac{\alpha}{\beta}\right]\right)=\left[\frac{\mathbf{x}_{c} \text {-sl }(\alpha)}{\beta}\right] \text { and } \mathrm{x}-\sup \left(\left[\frac{\alpha}{\beta}\right]\right)=\left[\frac{\mathbf{x}_{c} \text {-sup }(\alpha)}{\beta}\right] \text {, where } \\
& \text { a. } \operatorname{CAT}\left(\mathbf{x}_{c} \text {-sl }(\alpha)\right)=(A / C)[((B / C) \ddagger X) \\
& \text { b. } \operatorname{CAT}\left(\mathbf{x}_{c} \text {-sup }(\alpha)\right)=A^{C}\left[\left(B^{C} \ddagger X\right)\right. \\
& \text { c. } \llbracket \mathbf{x}_{c} \text {-sl }(\alpha) \rrbracket=\llbracket \mathbf{x}_{c} \text {-sup }(\alpha) \rrbracket=\lambda V_{\langle x, c b\rangle} \lambda C_{c} \cdot \llbracket \alpha \rrbracket\left(\lambda X_{x} \cdot V(X)(C)\right)
\end{aligned}
$$

In Figure 1, applying $x-s \mid$ to (a) converts the quantificational part of someone into a complex category that selects an $(\mathrm{S} / \mathrm{NP}) / \mathrm{NP}$ argument (corresponding to $\lambda \theta$ ). It also transmits the information about the 'trace' of everyone from the bottom component to the existential quantification (viz., passing up the abstraction of $x$ over so), which further forms the bottom component of the universal quantification. After the application of $\mathrm{x}-\mathrm{sl}$, inward FA can proceed as normal.

\subsubsection{Inverse scoping}

As mentioned in (29), in "Q1 V Q2", an inverse scope reading arises if it is the entire Q1 (as opposed to the 'trace'-part of Q1 only) that participates in composition as an argument of [V Q2]. A la Hendriks 1993, this condition can be achieved by argument-lift. In my system, argument-lift means lifting a $T$-category argument slot into one that needs to be filled by a two-dimensional quantifier, defined as in (31).

Argument lift (ALIFT; a la Hendriks 1993)

For any expression $\alpha$ s.t. $\operatorname{CAT}(\alpha)=(\mathrm{X} / \mathrm{NP}) \mid T$, we have:

$$
\operatorname{CAT}(\operatorname{ALifT}(\alpha))=\left[\frac{\Pi}{\mathrm{X} / \mathrm{NP}}\right] \mid\left[\frac{\Pi}{T}\right] \text { and } \llbracket \operatorname{ALift}(\alpha) \rrbracket=\lambda\left[\frac{\pi}{f_{e e}}\right] \cdot\left[\frac{\pi}{\lambda x_{e} \cdot \llbracket \alpha \rrbracket(f(x))}\right]
$$

Details of composition are given in Figure 2. First, by applying $\mathrm{ALift}_{1}$ to the VP, the subject argument slot which formerly called for a $T$ now wants to be filled by a two-dimensional quantifier. Second, composing this argument-lifted VP with the subject everyone by backward FA yields an inverse scope reading - so scopes above eo in (a). Finally, applying inward FA twice reduces the stack into a simple S. All the type-shifting and composition rules, except the last inward FA, carry the subscript 1, since they need to skip the extra slash-NP abstraction (corresponding to $\lambda y$ ) stemming from the 'trace'-part of the quantificational object someone. 
Binding without variables: solving the under-generation problems

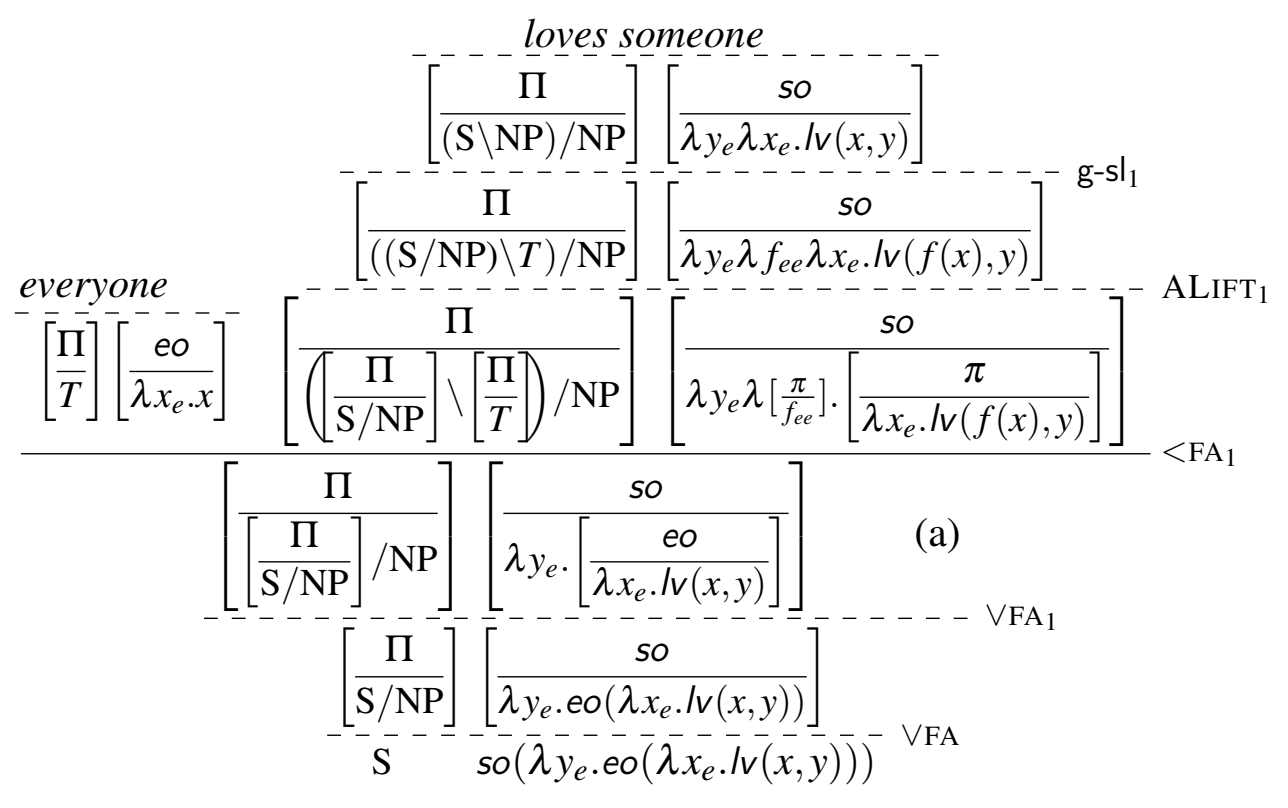

Figure 2 Inverse scope reading of Everyone loves someone

\subsection{Inverse linking}

Compared to the basic cases of inverse scoping, further complications arise in sentences with inverse linking. For example, the composition of (32) requires the following: (i) restrictor modification, to let the PP from every city restrict the quantification domain of someone; (ii) inverse scoping: to make every city take wide scope, and (iii) non-c-commanding binding: to allow every city to bind the pronoun it from a non-c-commanding position. Discussion in previous sections has explained (ii) and (iii), resorting to the applications of ALIFT and i, respectively. This subsection will add one more ingredient to derive restrictor modification.

(32) [Someone from every city $\left.i_{i}\right]$ [despises it $\left.i_{i}\right]$.

(May 1977)

(For every city $y$, there is an individual $x$ from $y$ s.t. $x$ despises $y$.)

Given the assumption that it is the 'trace'-part of someone (viz., a $T$-category denoting an identity function) that participates in composition, I define a restrictor modification rule as in (33). This rule converts a predicative modifier into a $T \backslash T$ category, which can modify 'traces'. (Here $f^{\prime} \leq f$ means that every pair in function $f^{\prime}$ is a pair in function $f$.)

(33) The RES-rule (to derive restrictor modification)

For any $P$ s.t. $\mathrm{CAT}(P)=\mathrm{S} \backslash \mathrm{NP}$, we have: $\operatorname{CAT}(\operatorname{RES}(P))=T \backslash T$, and 
$\llbracket \operatorname{RES}(P) \rrbracket=\lambda f_{e e} \cdot \operatorname{MAx}_{f^{\prime} \leq f}\left[\forall x^{\prime} \in \operatorname{Dom}\left(f^{\prime}\right)\left[\llbracket P \rrbracket\left(f^{\prime}\left(x^{\prime}\right)\right)\right]\right]$

(For any $f_{\langle e, e\rangle}$, return the maximal function $f^{\prime}$ that is a subpart of $f^{\prime}$ s.t. $f^{\prime}$ maps each object in its domain to an object satisfying $\llbracket P \rrbracket$.)

The complex subject in (32) is now composed as in Figure 3. Applying the restriction rule to from every city converts it into a restrictor of skolem functions. This restrictor undergoes argument-lift, so that it can apply to the entire two-dimensional expression someone and derive an inverse scope reading (every city 》someone).

Step (A): Composing the complex quantificational subject

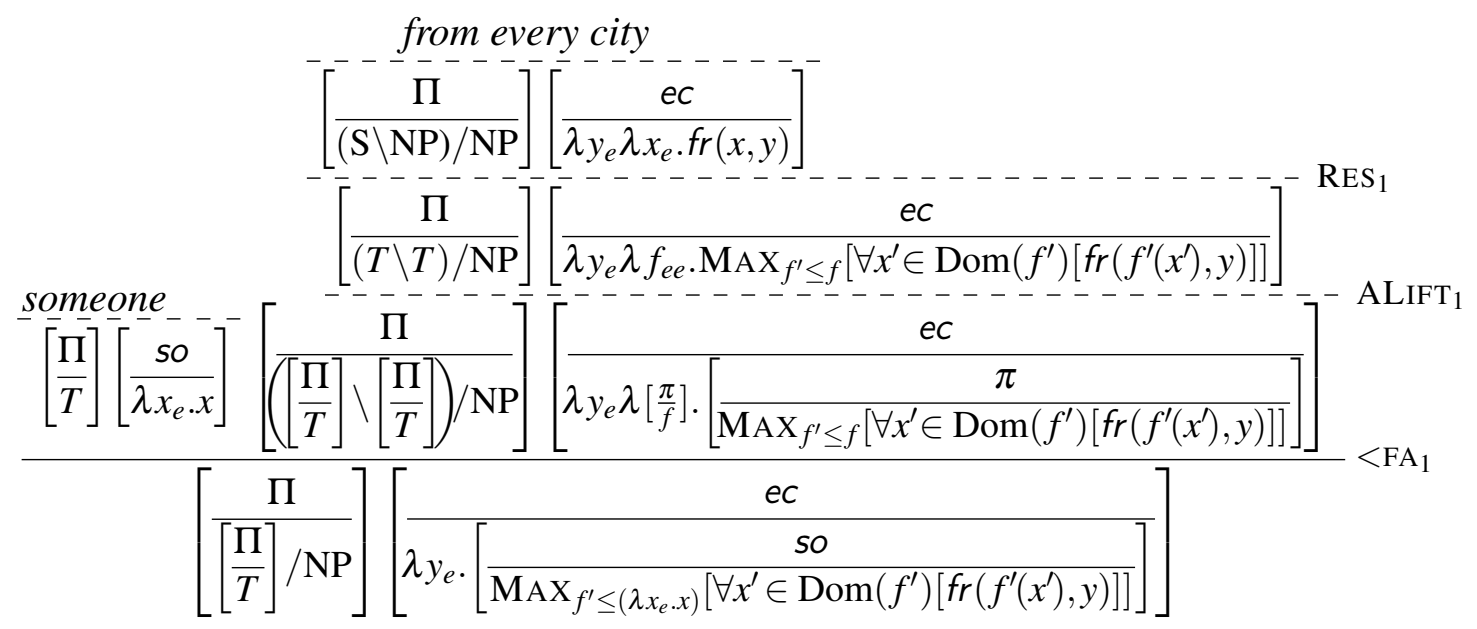

Figure 3 Composition of someone from every city (inverse scope reading)

In the composition output, the $T$-category part at the bottom is read as 'the maximal $\langle e, e\rangle$-type identity function $f^{\prime}$ that maps each entity in its domain to an entity from $y^{\prime}$. This $f^{\prime}$ has the same extension as the partial identity function $\lambda x_{e}: \operatorname{fr}(x, y) . x$. This step restricts the domain of some from $D_{e}$ to the set of entities from $y$.

The modified quantificational subject has a complex category. As illustrated in Figure 4: Step (B), to compose with this subject, the VP has to undertake a few of type-shifting operations till it selects an argument of category $\left[\frac{\Pi}{T}\right] / \mathrm{NP}$. In particular, the first $g$-sl ${ }_{1}$ and $\mathrm{ALIFT}_{1}$ are applied to allow the VP to select a two-dimensional quantificational argument, and the second $g$-s $\left.\right|_{1}$ is applied to let the VP to be ready to transmit the information about the 'trace' of every city in the subject.

The final steps of composition are given in Figure 4: Step (C). First, the complex subject composes with the type-shifted VP via backward FA. Next, applying the i-rule yields a dependency between the 'trace' of every city and the object pronoun it (corresponding to $\lambda y$ and $\lambda z$, respectively). Last, applying inward FA twice reduces the stack into a simple $\mathrm{S}$ expressing inverse linking. 
Binding without variables: solving the under-generation problems

Step (B): Type-shifting the VP

$$
\begin{aligned}
& \text { despises it }
\end{aligned}
$$

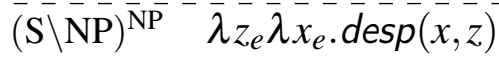

$$
\begin{aligned}
& \overline{((\mathrm{S} / \mathrm{NP}) \backslash T})^{\mathrm{NP}} \bar{\lambda}^{-} z_{e} \lambda f_{e e} \bar{\lambda} \bar{x}_{e} \cdot \overline{\operatorname{desp}}(\bar{f} \overline{(x)}, \bar{z})^{-\mathrm{g}-\mathrm{sl}}{ }_{1} \\
& \text { ( } \\
& \left(\left[\frac{\Pi}{\mathrm{S} / \mathrm{NP}}\right] \backslash\left[\frac{\Pi}{T}\right]\right)^{\mathrm{NP}} \quad \lambda z_{e} \lambda\left[\frac{\pi}{f}\right] \cdot\left[\frac{\pi}{\lambda x_{e} \cdot \operatorname{desp}(f(x), z)}\right] \\
& \left(\left(\left[\frac{\Pi}{\mathrm{S} / \mathrm{NP}}\right] / \mathrm{NP}\right) \backslash\left(\left[\frac{\Pi}{T}\right] / \mathrm{NP}\right)\right)^{\mathrm{NP}} \lambda z_{e} \lambda \rho_{\left\langle e,\left[\frac{\langle e t, t\rangle}{e e}\right]\right\rangle} \lambda y_{e} \cdot\left[\lambda\left[\frac{\pi}{f}\right] \cdot\left[\frac{\pi}{\lambda x_{e} \cdot \operatorname{desp}(f(x), z)}\right]\right](\rho(y))
\end{aligned}
$$

Step (C): Combining the complex subject with the VP

$$
\begin{aligned}
& \text { (A) someone from every city }
\end{aligned}
$$

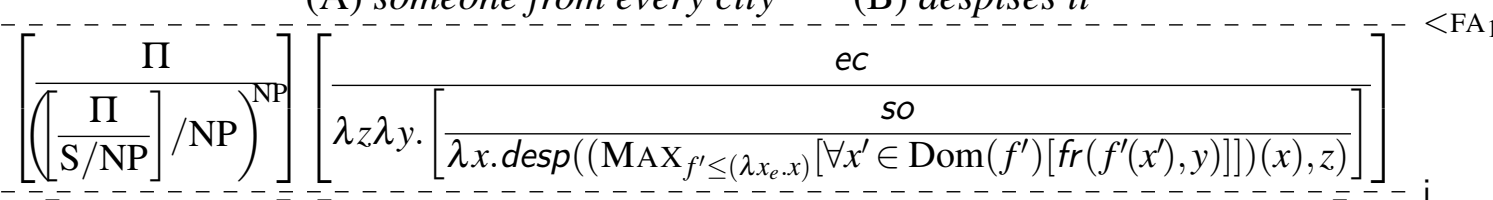

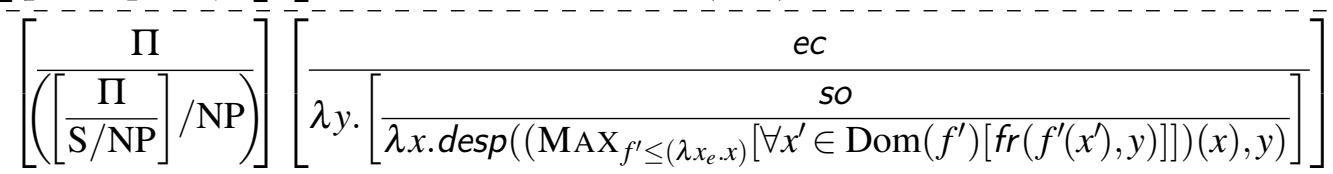

$$
\begin{aligned}
& {\left[\frac{\Pi}{\mathrm{S} / \mathrm{NP}}\right]\left[\frac{\mathrm{ec}}{\lambda y \cdot \operatorname{so}\left(\lambda x \cdot \operatorname{desp}\left(\left(\operatorname{MAX}_{f^{\prime} \leq\left(\lambda x_{e} \cdot x\right)}\left[\forall x^{\prime} \in \operatorname{Dom}\left(f^{\prime}\right)\left[\operatorname{fr}\left(f^{\prime}\left(x^{\prime}\right), y\right)\right]\right]\right)(x), y\right)\right)}\right]} \\
& {\left[\frac{\Pi}{\mathrm{S} / \mathrm{NP}}\right]\left[\frac{\mathrm{ec}}{\lambda y \cdot \operatorname{so}(\lambda x \cdot \operatorname{fr}(x, y) \wedge \operatorname{desp}(x, y))}\right]}
\end{aligned}
$$

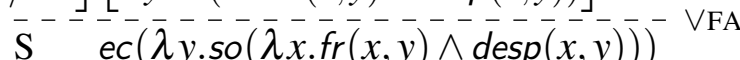

Figure 4 Composition of Someone from every city ${ }_{i}$ despises it ${ }_{i}$ (inverse linking)

\section{Weak crossover}

As well-known, scoping cannot feed binding in crossover constructions (Postal 1971; Chomsky 1976; a.o.). In (34), the pronominal possessor his in the subject cannot be bound by the quantificational object everyone. (For a review, see Safir 2017.)

His $_{j / * i}$ mom invited everyone ${ }_{i}$.

(Unavailable reading: 'For everyone $x, x$ 's mom invited $x$. ')

In my analysis, the deviance of weak crossover is straightforwardly predicted by the definedness constraint on the syntax of the i-rule. As defined in (13), the i-rule only applies to categories of the form ' $(\mathrm{X} ¥ \mathrm{NP})^{\mathrm{NP}}$. According to this constraint, the 
sup-NP abstraction provided by a bindable pronoun has to be reduced first, before any other NP abstraction saved for the binder of this pronoun is reduced.

According to this constraint, the i-rule can be applied to $(\mathrm{S} \backslash \mathrm{NP})^{\mathrm{NP}},(\mathrm{S} / \mathrm{NP})^{\mathrm{NP}}$, and $\left(S^{N P}\right)^{N P}$, as exemplified in $(36 \mathrm{a}-\mathrm{c})$. In these three cases, the NP abstraction for the binder comes from an unsaturated syntactic argument of loves, the 'trace'-part of the two-dimensional quantifier every boy, and the pronoun his, respectively.
a. i [loves him]
b. i [every boy's mom loves him]
c. i [his mom loves him]

In contrast, the i-rule cannot be applied in (34), neither to the VP nor to the full sentence. As seen below, both strings have an (S\$NP)/NP-category at the bottom. The composition in (36) yields an $\mathrm{S}^{\mathrm{NP}}$ that expresses an unbound reading.

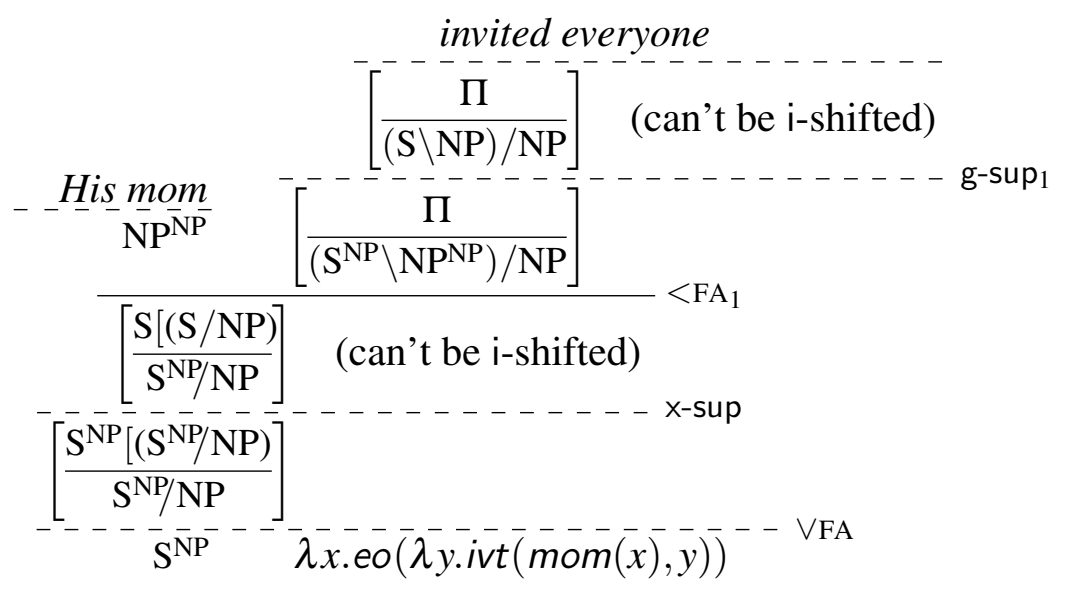

\section{Paycheck pronouns}

In the following sentences, the pronouns in bold are called 'paycheck pronouns' (Karttunen 1969; Cooper 1997; Engdahl 1986; Jacobson 2000; Barker 2005; a.o.). In (37a), her refers to Billy's mom. Descriptively, this reading arises as follows: her refers to the function his mom, in which the possessive pronoun his is bound by Billy. The same applies to the pronoun it in (37b) (example from Jacobson 2000).

a. Andy loves his $_{i}$ mom, but Billy ${ }_{j}$ hates her $=$ his $_{j}$ mom. (Intended: 'Andy loves his mom, but Billy hates his mom.')

b. The woman [who ${ }_{i}$ deposited her ${ }_{i}$ paycheck in the bank] was wiser than the woman [ who $_{j}$ deposited $\overline{\mathbf{i t}_{=\text {her }_{j} \text { paycheck }}}$ in the Credit Union].

Jacobson's variable-free semantics naturally accounts for the functional interpretations of paycheck pronouns: first, her in lexicon is an $\mathrm{NP}^{\mathrm{NP}}$-category denoting 
an identify function over individuals; second, applying g-sup to her turns it into an $\left(\mathrm{NP}^{\mathrm{NP}}\right)^{(\mathrm{NPNP})}$-category that denotes an identity function over $\langle e, e\rangle$-type functions, such as $\llbracket$ his $m o m \rrbracket\left(=\lambda x_{e} \cdot \operatorname{mom}(x)\right)$. This merit of Jacobson's system is also manifested in analyzing Bach-Peters sentences (Jacobson 2000) as well as wh-questions and $w h$ - relative clauses with functional interpretations (Jacobson 1999).

However, Jacobson's system doesn't generate non-c-commanding binding, which includes not only aforementioned possessor binding but also cross-sentential binding. Jacobson (2000) argues that in (37a) the co-referential relation between his mom and the paycheck pronoun her isn't really a binding relation; instead, she treats her as a free pronoun, which picks up the contextually salient value denoted by his mom.

In contrast, the proposed system allows us to derive a cross-sentential dependency between his mom and her. By lifting $\mathrm{NP}^{\mathrm{NP}}$-categories into two-dimensional quantifiers, the analysis made for non-c-commanding quantificational binding also extends to the binding of a paycheck pronoun. I generalize the Montague lift rule as in (38), which works cross-categorically for NPs as well any pronominal categories.

(38) Cross-categorical lift (generalized from (27))

For any X-category $\alpha$ s.t. X is NP or a pronominal category, we have:

$\operatorname{CAT}(\operatorname{LifT}(\alpha))=\left[\frac{\mathrm{S}[(\mathrm{S} / \mathrm{X})}{\mathrm{X} / \mathrm{X}}\right]$, and $\llbracket \operatorname{LiFT}(\alpha) \rrbracket=\left[\frac{\lambda P_{\langle x, t\rangle} \cdot P(\llbracket \alpha \rrbracket)}{\lambda X_{x} \cdot X}\right]$

(39) Pronominal categories (after Charlow To appear)

$\mathrm{NP}^{\mathrm{NP}}$ is a pronominal category; if $\mathrm{Y}^{\mathrm{X}}$ is a pronominal category, $\left(\mathrm{Y}^{\mathrm{NP}}\right)^{\left(\mathrm{X}^{\mathrm{NP}}\right)}$ is a pronominal category; nothing else is a pronominal category.

The composition of (37a) is given in (40), which omits the g-rules applied for resolving type-mismatch. This composition includes the following operations: (i) as assumed by Jacobson (2000), applying g-sup to the paycheck pronoun her allows this pronoun to refer to an $\langle e, e\rangle$-type function such as his mom (as opposed to an individual); (ii) in both clauses, applying i to VP yields a dependency between the subject and the pronominal object in each clause; (iii) together with the results of operation (i), lifting his mom and applying i to the entire coordination make her be bound by his mom. This analysis also applies to (37b).

$$
\begin{aligned}
& \text { [Andy i [loves LIFT(his mom)]] }
\end{aligned}
$$

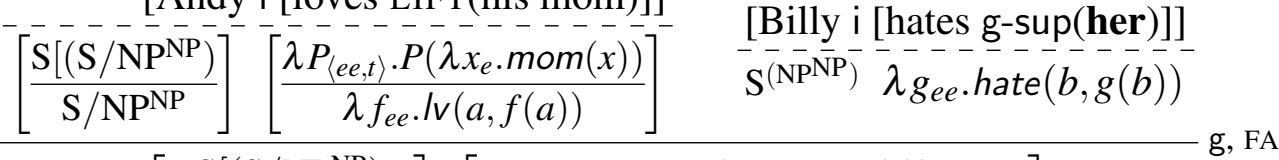

$$
\begin{aligned}
& {\left[\frac{\mathrm{S}\left[\left(\mathrm{S} / \mathrm{NP}^{\mathrm{NP}}\right)\right.}{\left(\mathrm{S} / \mathrm{NP}{ }^{\mathrm{NP}}\right)^{(\mathrm{NPNP})}}\right]\left[\frac{\lambda P_{\langle e e, t\rangle} \cdot P\left(\lambda x_{e} \cdot \operatorname{mom}(x)\right)}{\lambda g_{e e} \lambda f_{e e} \cdot / v(a, f(a)) \wedge \text { hate }(b, g(b))}\right]} \\
& {\left[\frac{\mathrm{S}[(\mathrm{S} / \mathrm{NPNP})}{\mathrm{S} / \mathrm{NPNP}}\right]\left[\frac{\lambda P_{\langle e e, t\rangle} \cdot P\left(\lambda x_{e} \cdot \operatorname{mom}(x)\right)}{\lambda f_{e e} \cdot I v(a, f(a)) \wedge \operatorname{hate}(b, f(b))}\right]} \\
& \operatorname{lv}(\bar{a}, \operatorname{mom}(\bar{a})) \wedge \text { hate }(\bar{b}, \operatorname{mom}(b))
\end{aligned}
$$




\section{Comparison with Barker (2005)}

To account for 'non-c-commanding' binding, Barker (2005) enriches the variablefree system of Jacobson 1999 by assuming a separate pair of rules, called s and q. As exemplified in (42), the s-rule, defined as a dual of $z$, allows a quantificational binder to bind one level up. Composition with quantifiers is achieved by value raising (VR) and argument lift (ALIFT). In cases where the binder is deeply embedded, the q-rule (cf. g) transmits the information about this binder (details are omitted).

\section{The s-rule}

For any expression $\alpha$ s.t. $\operatorname{CAT}(\alpha)=(A \mid B) \mid \mathrm{NP}$, we have:

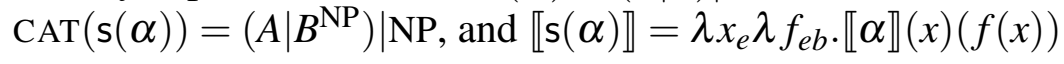

Every boy ${ }_{i}$ 's mom loves $\operatorname{him}_{i}$. $(G$ abbreviates $\mathrm{S} /(\mathrm{S} \backslash \mathrm{NP}))$

$$
\begin{aligned}
& \text { 's mom } \\
& \mathrm{NP} \backslash \mathrm{NP} \quad \lambda x \cdot \operatorname{mom}(x) \\
& (\overline{\mathrm{S}} /(\overline{\mathrm{S}} \backslash \overline{\mathrm{NP}})) \backslash \overline{\mathrm{NP}}-\bar{\lambda} \bar{x} \bar{\lambda} \bar{\pi} \cdot \bar{\pi}(\bar{\lambda} \bar{P} \cdot \bar{P}(\operatorname{mom}(\bar{x})))^{-\mathrm{VR}}
\end{aligned}
$$

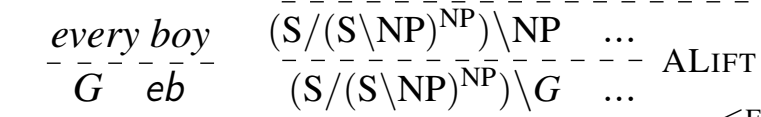

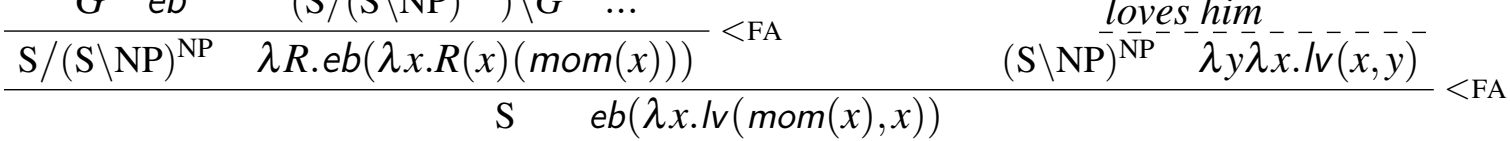

The s-rule, however, is abandoned in Jacobson 1999 since it allows for crossover. To avoid generating crossover constructions, Barker further builds a separate value assigning and transmitting system.

In comparison, while Barker (2005) makes use of two binding rules, i.e., z and s, my analysis needs only one binding rule, i.e., the i-rule. In my account, crossover constructions are automatically ruled out by a syntactic constraint on the application of i. Further, by defining quantificational binders as two-dimensional expressions with a 'trace'-like NP/NP-component, my account captures the transmitting of the information about the binder by the existing rule g-sl and doesn't require rules like q.

\section{Conclusion}

This paper presented a solution to the binding under-generation problems of Jacobson's variable-free system. I argued to derive dependencies uniformly by the i-rule, which is more flexible than Jacobson's locally applied z-rule. I also argued to analyze quantifiers as two-dimensional expressions, which consist of a quantificational part and a 'trace'-like part. This two-dimensional treatment is particularly useful for tackling scoping and 'non-c-commanding' binding. In future research, I would like to see how the enriched system compares to competing variable-free approaches to binding (Szabolcsi 1992; Barker \& Shan 2014; Charlow To appear; a.o.). 
Binding without variables: solving the under-generation problems

\section{References}

Barker, Chris. 2005. Remark on Jacobson 1999: Crossover as a local constraint. Linguistics and Philosophy 28(4). 447-472. doi:10.1007/s10988-004-5327-1.

Barker, Chris. 2012. Quantificational binding does not require c-command. Linguistic Inquiry 43(4). 614-633. doi:10.1162/ling_a_00108.

Barker, Chris \& Pauline Jacobson. 2007. Introduction: Direct compositionality. In Chris Barker \& Pauline Jacobson (eds.), Direct compositionality, 1-19. Oxford: Oxford University Press.

Barker, Chris \& Chung-chieh Shan. 2014. Continuations and natural language. Oxford: Oxford University Press.

Charlow, Simon. To appear. Variable-free semantics and flexible grammars for anaphora. Studies in Linguistics and Philosophy 100. https://ling.auf.net/ lingbuzz/004503.

Chomsky, Noam. 1976. Constraints on rules of grammar. Linguistic Analysis 2. 303-351.

Cooper, Robin. 1983. Quantification and syntactic theory. Netherlands: Springer. doi:10.1007/978-94-015-6932-3.

Cooper, Robin. 1997. The interpretation of pronouns. In Frank Heny \& Helmut S. Schnelle (eds.), Selections from the third Groningen round table, 61-92. New York: Academic Pres.

Engdahl, Elisabet. 1986. Constituent questions: The syntax and semantics of questions with special reference to Swedish, vol. 27. Dordrecht: Reidel. doi:10.1007/978-94-009-5323-9.

Geach, Peter Thomas. 1970. A program for syntax. Synthese 22. 3-17. doi:10.1007/BF00413597.

Hendriks, Herman. 1993. Studied Flexibility: Categories and types in syntax and semantics: Institute for Logic, Language and Computation, University van Amsterdam $\mathrm{PhD}$ dissertation.

Jacobson, Pauline. 1999. Towards a variable-free semantics. Linguistics and Philosophy 22(2). 117-185. doi:10.1023/A:1005464228727.

Jacobson, Pauline. 2000. Paycheck pronouns, Bach-Peters sentences, and variable-free semantics. Natural Language Semantics 8(2). 77-155. doi:10.1023/A:1026517717879.

Jacobson, Pauline. 2014. Compositional semantics: An introduction to the syntax/semantics interface. Oxford: Oxford University Press.

Karttunen, Lauri. 1969. Pronouns and variables. In Robert I. Binnick, Alice Davison, Georgia M. Green \& Jerry L. Morgan (eds.), Proceedings from the Annual Meeting of the Chicago Linguistic Society, vol. 5 1, 108-116. Chicago Linguistic Society. 
Kratzer, Angelika \& Irene Heim. 1998. Semantics in generative grammar. Oxford: Wiley-Blackwell.

May, Robert. 1977. The Grammar of Quantification: MIT PhD dissertation.

Postal, Paul Martin. 1971. Cross-over phenomena. New York: Holt, Rinehart and Winston.

Safir, Ken. 2004. The syntax of (in) dependence. Cambridge, MA: MIT Press. doi:10.7551/mitpress/6595.001.0001.

Safir, Ken. 2017. Weak crossover. In M. Everaert \& H. van Riemsdijk (eds.), The Wiley Blackwell companion to syntax, second edition, 1-40. New York: Wiley. doi:10.1002/9781118358733.wbsyncom090.

Shan, Chung-chieh \& Chris Barker. 2006. Explaining crossover and superiority as left-to-right evaluation. Linguistics and Philosophy 29(1). 91-134. doi:10.1007/s10988-005-6580-7.

Szabolcsi, Anna. 1992. Combinatory grammar and projection from the lexicon. In Ivan Sag \& Anna Szabolcsi (eds.), Lexical matters, 241-268. Stanford: CSLI publications.

Yimei Xiang

18 Seminary Place, Room 205

Department of Linguistics

Rutgers University

New Brunswick, NJ 08901-1184

USA

yimei.xiang@ rutgers.edu 